\title{
A retrospective study on prevalence, bacteriological profile and antibiotic sensitivity pattern of urinary tract Infection in children of 2- 12 years age in a tertiary care centre, Puducherry, India
}

\author{
Dharmalingam Angamuthu, Chandru Bhaskar*, Nalini Aswathaman
}

Department of Paediatrics, Aarupadai Veedu Medical College and Hospital, Kirumampakkam, Puducherry, India

Received: 12 June 2019

Accepted: 17 July 2019

\author{
*Correspondence: \\ Dr. Chandru Bhaskar, \\ E-mail: Chandru14885@gmail.in
}

Copyright: (C) the author(s), publisher and licensee Medip Academy. This is an open-access article distributed under the terms of the Creative Commons Attribution Non-Commercial License, which permits unrestricted non-commercial use, distribution, and reproduction in any medium, provided the original work is properly cited.

\begin{abstract}
Background: Urinary tract infections (UTIs) are a common cause of acute bacterial illness in infants and children(1).Diagnosis is often missed in infants and children as urinary symptoms are minimal and nonspecific. It is becoming increasingly difficult to treat due to increasing prevalence of antimicrobial resistance among organisms.

Method: It was done as a retrospective cross sectional study conducted on Children of 2-12 years of age suspected to have UTI from January 2018 to December 2018 in Pediatric Department of AVMCH.

Results: Out of 286 children with symptoms suggestive of Urinary tract infection, 22(7.7\%) were found to have culture positive UTI with $9.2 \%$ prevalence in 2-6 years age group as against $6.4 \%$ in 7-12 years of age group. Culture positive UTI was predominantly found in males in 2-6 years age group as against female predominance in 7-12 years age group. E. coli $(45.5 \%)$ was the commonest organism isolated among them followed by Klebsiella. $90 \%$ of organisms were sensitive to Nitrofurantoin and $80 \%$ were sensitive to Amikacin( $80 \%)$.

Discussion: Culture positive UTI rate varies with gender and age group. It was predominantly found in males in 2-6 years age group as against female predominance in 7-12 years age group. E. coli and Klebsieella were the most common organisms with predominant sensitivity to Nitrofurantoin and Amikacin similar to other studies. Limitation of this study Retrospective study, small sample size and follow up for complications not done.

Conclusion: This study concludes E. coli and Klebsieella were the most common organisms with predominant sensitivity to Nitrofurantoin and Amikacin. Antibiotics need to be selected based on organisms and their sensitivity pattern.
\end{abstract}

Keywords: Antibiotic sensitivity, Prevalence, Urinary tract infection

\section{INTRODUCTION}

Urinary tract infections (UTIs) are a common cause of acute bacterial illness in infants and children. ${ }^{1}$ Urinary Tract Infection is defined as the microbial invasion of any of the tissue of the urinary tract extending from the renal cortex to the urethral meatus. Urine formed in the kidney is a sterile fluid that serves as a good culture medium for proliferation of bacteria. ${ }^{2}$ The diagnosis of UTI is often missed in infants and children, as urinary symptoms are minimal and nonspecific. Timely diagnosis and adequate, appropriate antibiotic treatment is necessary to prevent renal damage in the form of renal scarring, hypertension and subsequent renal parenchymal damage. It is an important cause of fever without focus in children and presents as a part of septicaemia with fever, vomiting, lethargy, Jaundice and seizures. Older children are able to verbalize better specific symptoms, such as dysuria, frequency, urgency, new onset urinary incontinence, 
abdominal or flank pain, suprapubic discomfort, and vomiting.

Acute UTIs are relatively common in children. By seven years of age, 8 percent of girls and 2 percent of boys will have at least one episode. After preliminary cleaning of the genitalia, clean catch mid-stream urine should be collected by caregiver and subjected for laboratory analysis. Diagnosis of urinary tract infection is by growth of a significant number of organisms of a single species in the urine, in the presence of symptoms. ${ }^{3}$ Combination of clinical symptoms, pyuria and positive urine culture with $>50,000 \mathrm{CFU} / \mathrm{ml}$ of a single pathogenic organism on an appropriately collected urine culture. ${ }^{4}$ The reference standard for the diagnosis of UTI is a single organism cultured from a specimen obtained at the following concentrations: supra pubic aspiration specimen-greater than 1,000 colony-forming units per $\mathrm{ml}$; catheter specimen- greater than 10,000 colony-forming units per $\mathrm{ml}$; or midstream clean-catchspecimen-100,000 colonyforming units per $\mathrm{ml}$ or greater. ${ }^{5}$ In older children, sample for urinalysis and urine culture can be collected from suprapubic aspiration or urethral catheterization when the child is ill and require immediate antibiotic therapy.

Common uropathogens include Escherichia coli (accounting for approximately 85 percent of UTIs in children), Klebsiella, Proteus, Enterobacter, Citrobacter, Staphylococcus saprophyticus, and Enterococcus. ${ }^{6}$ Biochemical analyses of leukocyte esterase and nitrite through a rapid dipstick method and urine microscopic examination for pyuria and bacteriuria are carried out. The combination of both leukocyte esterase and nitrite tests is highly sensitive and specific. Recent study shows dipstick may be an adequate screening test for UTI with a negative predictive value of $98.7 \%$. As it is standard procedure, adding microscopy increases the negative predictive value to $99.2 \%$ but results in 8 false-positive tests for every UTI missed by dipstick. ${ }^{7,8}$

Objective of this study was to prevalence of culture positive urinary tract infection in children of 2-12 years age and its bacteriological profile with antibiotic sensitivity pattern over one year period.

\section{METHODS}

This retrospective cross sectional study was done after Institutional Ethical committee clearance in 286 children of 2-12 years age group with suspected UTI infection who attended Paediatric OPD and admitted in wards of AVMC and H from January 2018 to December 2018. A brief clinical history obtained from each patient and their parents' to find out if there was any risk factor predisposing to UTI. After cleaning genitalia with water, mid-stream clean catch urine was collected and subjected for laboratory analysis. Urine samples were examined and processed in the laboratory as soon as possible after collection.

\section{Microscopy}

Urine specimens examined by wet mounts and gram staining. Presence of any pus cells, microorganisms, RBC's, cast and crystals or any other findings noted. Organisms grown in urinary culture growth were identified by relevant biochemical reactions.

\section{Culture}

Urine specimen was plated on blood agar and MacConkey agar. After 24 hours of incubation, culture plates were observed for growth and bacterial species identified. Antibiotic sensitivity testing. In the presence of any potential growth, antibiotic sensitivity testing were done by the modified Kirby-Bauer disc diffusion method according to the Clinical and Laboratory Standards Institute (CLSI) guidelines. The antibiotics tested were Nitrofurantoin, Amikacin, Ciprofloxacin, Cotrimoxozole, Amoxycillin Clavulanate, Piperacillin, Linezolid, Nalidixic Acid, Streptomycin and Gentamicin.

\section{Analysis}

Data were collected and entered in Microsoft excel 2010. It was analysed using frequency distribution in number and percentage.

\section{RESULTS}

Overall $7.7 \%$ of children with suspected UTI had culture positive sepsis with more prevalence (9.2\%) among 2-6 years age as compared to 7-12 years age.54.5\% were males as against $45.5 \%$ of females in our study. 2 to 6 years age group had higher prevalence $(9.2 \%)$ of culture positive UTI as against only $6.4 \%$ in 7 to 12 years age group. Prevalence of Culture positive UTI in the 2 to 6 years age group were $10.8 \%$ and $7.1 \%$ in males and females respectively. In the age group 7 to 12 years, Prevalence of culture positive UTI were $5.9 \%$ and $6.9 \%$ in males and females respectively. Culture positive UTI was predominantly found in males in 2-6 years age group as against female predominance in 7-12 years age group. (Table 1).

E. coli $(45.5 \%)$ was the commonest organism isolated among them in our study followed by Klebsiella which is $18.1 \% .2 .8 \%$ among the suspected UTI had mixed growth of organisms and hence discarded from the analysis (Table 2). E. coli had sex predilection of male in 2-6 years age as compared Female predilection in 7-12 years age group (Table 3 ). Only females in 2-6 years age group had been affected with Klebsiella urinary tract infections as against no sex predilection in 7-12 years age group (Table 4). All E. coli were susceptible to Nitrofurantoin and Amikacin but developing resistance to other antibiotics. (Figure 3). All Klebsiella were sensitive to Nitrofurantoin and all of them were resistant for Amoxycillin-clavulanate, Ciprofloxacin and Carbapenem 
(Figure 4). $90 \%$ of organisms were sensitive to Nitrofurantoin followed by Amikacin (80\%) (Table 5).

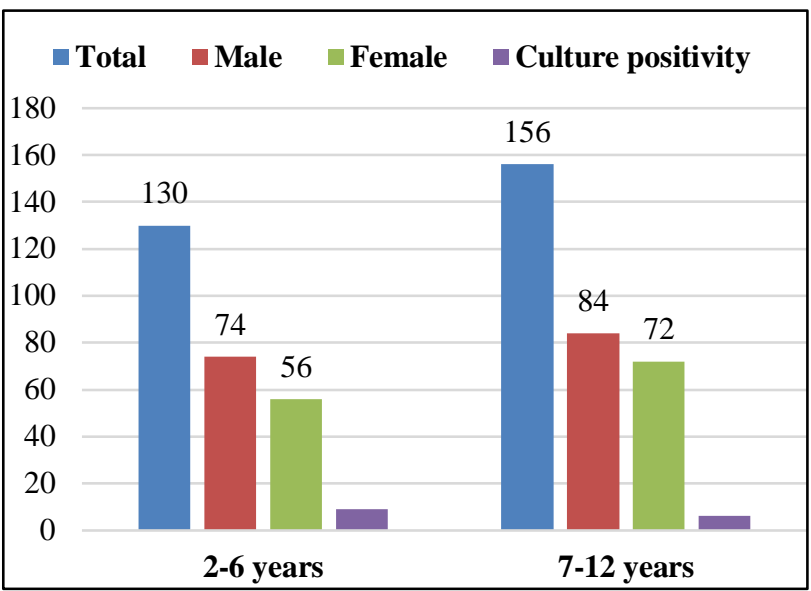

Figure 1: Age and Sex distribution in relation to positive urine culture.

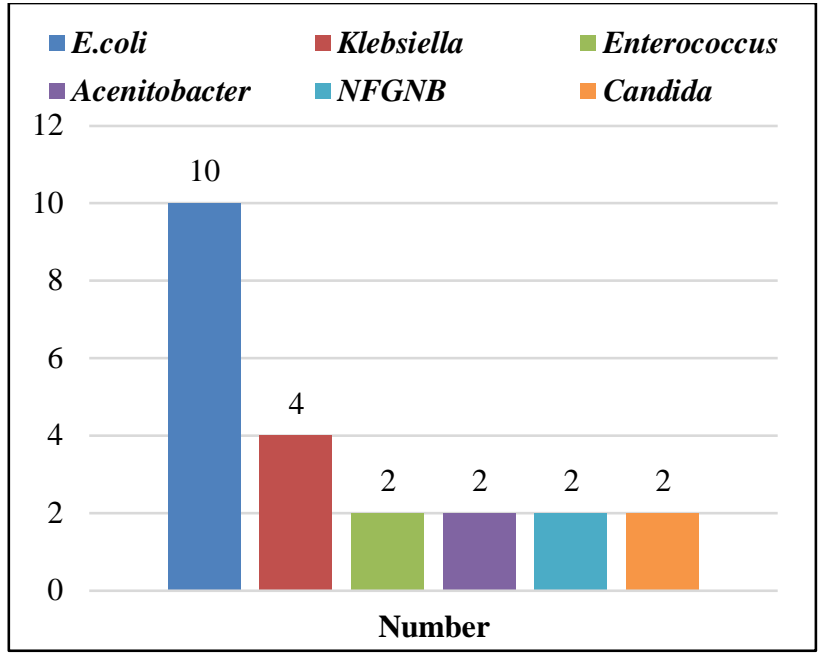

Figure 2: Bacteriological profile of urinary tract infections.

Table 1: Age and sex distribution in relation to positive urine culture.

\begin{tabular}{|lllllll|}
\hline Age Group & $\begin{array}{l}\text { Total no. of } \\
\text { patients }\end{array}$ & Male & Female & $\begin{array}{l}\text { Total positive } \\
\text { culture }\end{array}$ & $\begin{array}{l}\text { Positive } \\
\text { culture males }\end{array}$ & $\begin{array}{l}\text { Positive culture } \\
\text { females }\end{array}$ \\
\hline 2 to 6 Years & $130(48.9 \%)$ & 74 & 56 & $12(9.2 \%)$ & $8(10.8 \%)$ & $4(7.1 \%)$ \\
\hline 7 to 12 Years & $156(51.1 \%)$ & 84 & 72 & $10(6.4 \%)$ & $5(5.9 \%)$ & $5(6.9 \%)$ \\
\hline
\end{tabular}

Table 2: Bacteriological profile of urinary tract infections.

\begin{tabular}{|lll|lll|}
\hline E. coli & Klebsiella & Enterococci & Acinetobacter & Non Fermenting Gram Negative Bacteria & Candida \\
\hline $10(45.5 \%)$ & $4(18.1 \%)$ & $2(9.1 \%)$ & $2(9.1 \%)$ & $2(9.1 \%)$ & $2(9.1 \%)$ \\
\hline
\end{tabular}

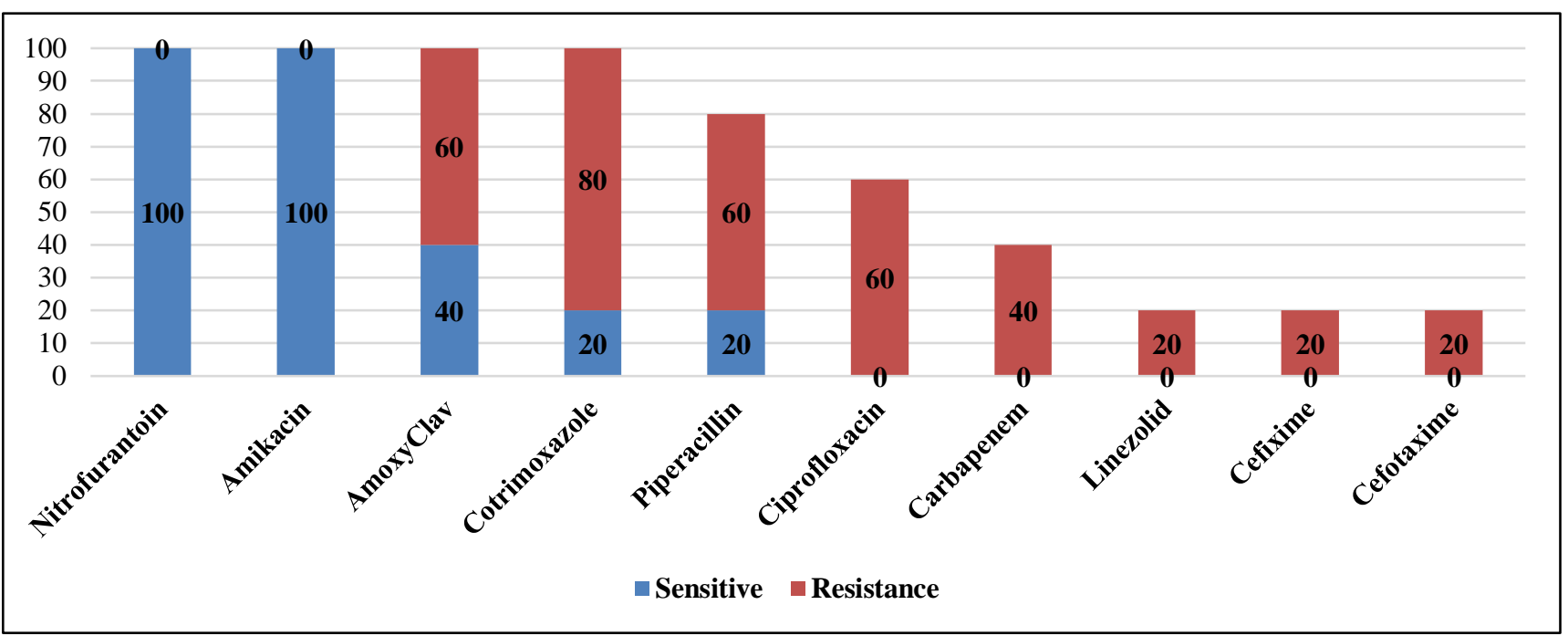

Figure 3: Antibiotic sensitivity pattern among $E$. coli sepsis.

Table 3: Sensitivity pattern of $E$. coli according to age group.

\begin{tabular}{|lll|}
\hline Age Group & Male & Female \\
\hline 2 to 6 Years & $5(50 \%)$ & $1(10 \%)$ \\
\hline
\end{tabular}

Table 4: Sensitivity pattern of Klebsiella according to age group.

\begin{tabular}{|lll|}
\hline Age Group & Male & Female \\
\hline 2 to 6 Years & $0(0 \%)$ & $2(50 \%)$ \\
\hline 7 to 12 Years & $1(25 \%)$ & $1(25 \%)$ \\
\hline
\end{tabular}




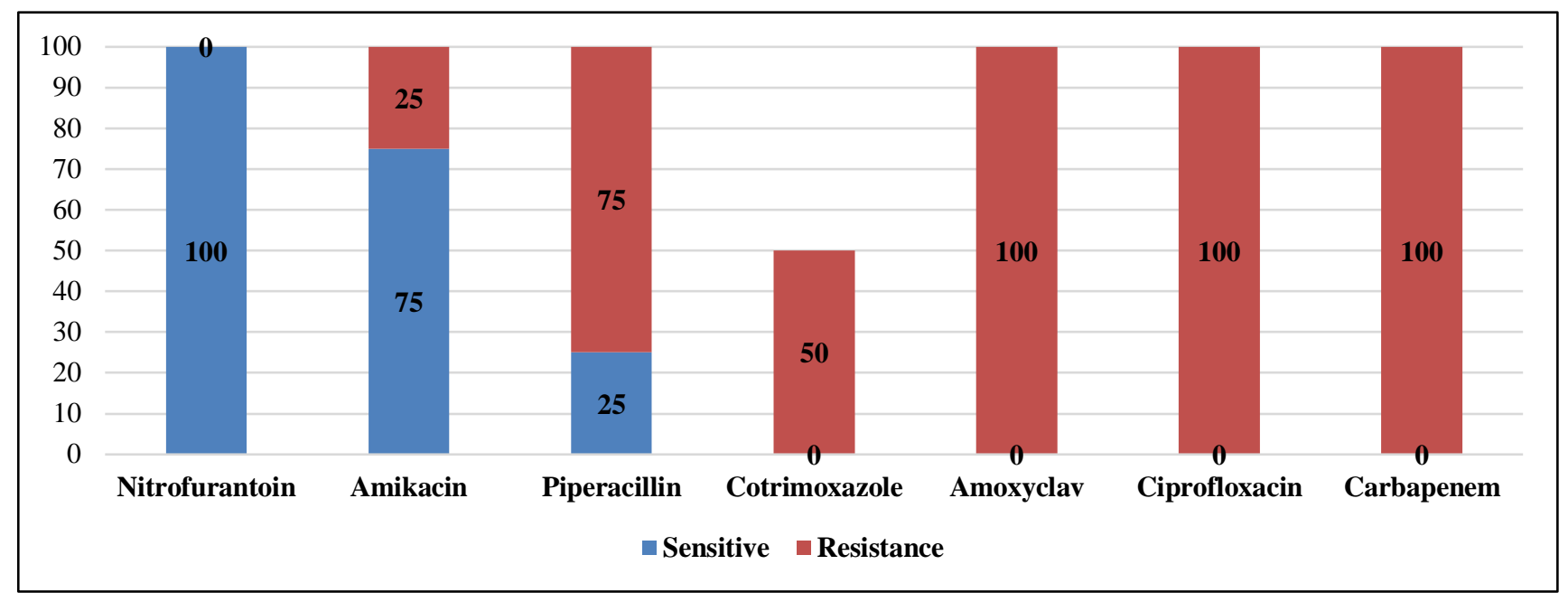

Figure 4: Antibiotics susceptibility pattern among Klebsiella.

Table 5: Overall antibiotic sensitivity pattern of all organisms together.

\begin{tabular}{|l|ll|}
\hline S. no. & Antibiotics & $\begin{array}{l}\text { Percentage } \\
\text { sensitive }\end{array}$ \\
\hline 1 & Nitrofurantoin & $90 \%$ \\
\hline 2 & Amikacin & $80 \%$ \\
\hline 3 & Ciprofloxacin & $25 \%$ \\
\hline 4 & Cotrimoxozole & $20 \%$ \\
\hline 5 & Amoxycillin Clavulanate & $20 \%$ \\
\hline 6 & Piperacillin & $15 \%$ \\
\hline 7 & Linezolid & $10 \%$ \\
\hline 8 & Nalidixic Acid & $10 \%$ \\
\hline 9 & High Level Gentamicin & $5 \%$ \\
\hline 10 & Streptomycin & $5 \%$ \\
\hline
\end{tabular}

\section{DISCUSSION}

Prevalence of culture proven UTI was 7.7\% in our study which was much lower than Palak et al and Iranian study in which $35.4 \%$ and $34.2 \%$ of suspected UTI children had Culture proven UTI respectively. ${ }^{9-10}$ Culture positive rate was $15.7 \%, 26.8 \%$ and $18.3 \%$ in other studies by Kaur, Thaddenee and Patwardhan respectively. ${ }^{11-13}$ This wide difference may be due to infant being the major contributor of culture positive UTI in Kaur study and we have taken only from 2-12 years age group. Males were predominant in our study in both age groups which may explain gender preference of health seeking behaviour. In age group of 2-6 years males and in 7-12 years females predominantly had prevalence was of culture positive UTI similar to other studies. In the age group of 2 to 6 years the prevalence is $9.2 \%$ and $6.4 \%$ were in the age group of 7 to 12 years which may be due to improvement in their immunity and personal hygiene which could be contributing to lower prevalence in 7-12 years age group.
Prevalence of Culture positive UTI in the 2 to 6 years age group were $10.8 \%$ and $7.1 \%$ in males and females respectively similar to Taneja study of higher prevalence in males. ${ }^{14}$ In the age group 7 to 12 years, Prevalence of culture positive UTI were $5.9 \%$ and $6.9 \%$ in males and females respectively. Culture positive UTI was predominantly found in males in 2-6 years age group as against female predominance in 7-12 years age group. $E$. coli $(45.5 \%)$ was the commonest organism isolated among them in our study similar to Kaur et al of $45.4 \%$ and Kulkarni study. ${ }^{15}$ Next most common organism isolated is Klebsiella which is $18.1 \%$ in our study versus $16.1 \%$ in Kaur study. ${ }^{11}$ In Iranian study, E.coli was isolated in $57.4 \%$ among the culture positivity. ${ }^{9} 90 \%$ of organisms were sensitive to Nitrofurantoin as against $51.8 \%$ in Iranian study, 60\% in Patwardhan study and $75.8 \%$ in a systematic review by Ashley. ${ }^{12,16}$ Sensitivity of amikacin in this study was $80 \%$ as against Mashouf study of $93.9 \%$.These antibiotics resistance pattern can persist for 6 months after treatment. Hence oral nitrofurantoin can be started which would be cheap, sensitive antibiotics and reduce direct and indirect cost of hospitalization.

Retrospective study, small sample size and follow up for complications not done; were the limitations of this study.

\section{CONCLUSION}

The optimal diagnosis of UTI in children should be based on clinical presentation, physical examination, urinalysis interpretation, methods of urine collection, cut off threshold of urine culture, inflammatory markers, and sometimes imaging studies. Timely and appropriate diagnosis and prompt treatment of UTI is important to eradicate acute infection, prevent bacteremia, improve 
clinical condition and reduce the likelihood of renal damage. E. coli and Klebsieella were the most common organisms with predominant sensitivity to Nitrofurantoin and Amikacin in our study. Empirical Antibiotics need to be selected based on organisms and their sensitivity pattern in that region.

Funding: No funding sources

Conflict of interest: None declared

Ethical approval: The study was approved by the Institutional Ethics Committee

\section{REFERENCES}

1. Robinson JL, Finlay JC, Lang ME, Bortolussi R. Urinary tract infections in infants and children: Diagnosis and management. Paediatr Child Health. 2014;19(6):315-9.

2. Prevalence of Urinary Tract Infection Among Pregnant Women and its Complications in Their Newborns During the Birth in the Hospitals of Dezful City, Iran, 2012-2013 cited 2019. Available at:

https://www.ncbi.nlm.nih.gov/pmc/articles/PMC458 5427/ Accessed 5 June 2019.

3. Vijayakumar M, Kanitkar M, Nammalwar BR, Bagga A. Revised statement on management of urinary tract infections. Indian pediatr. 2011 Sep;48(9):709-17.

4. Consensus Guidelines for Management of Pediatric Urinary Tract Infection (UTI): Northern California Pediatric Hospital Medicine Consortium 2019. Available at: https://www.ucsfbenioffchildrens.org/pdf/urinarytract-infection-full-guideline.pdf Accessed 5 June 2019.

5. Stein R, Dogan HS, Hoebeke P, Kočvara R, Nijman RJM, Radmayr C, et al. Urinary tract infections in children: EAU/ESPU guidelines. Eur Urol. 2015 Mar;67(3):546-58.

6. White B. Diagnosis and treatment of urinary tract infections in children. American family physician. 2011Feb15;83(4)7.

7. Simões e Silva AC, Oliveira EA. Update on the approach of urinary tract infection in childhood. Jornal de Pediatria (Versãoem Português). 2015 Nov;91(6):S2-10.

8. Zorc JJ, Kiddoo DA, Shaw KN. Diagnosis and Management of Pediatric Urinary Tract Infections. Clin Microbiol Reviews. 2005Apr1;18(2):417-22.

9. Mashouf RY, Babalhavaeji H, Yousef J. Urinary tract infections: bacteriology and antibiotic resistance patterns. Indian pediatrics. 2009 Jul $1 ; 46(7)$.

10. Gupta P, Mandal J, Krishnamurthy S, Barathi D, Pandit N. Profile of urinary tract infections in paediatric patients. Ind J Medical Research. 2015 Apr 1;141(4):473.

11. Kaur N, Sharma S, Malhotra S, Madan P, Hans C. Urinary Tract Infection: Aetiology and Antimicrobial Resistance Pattern in Infants from A Tertiary Care Hospital in Northern India. J Clin Diagn Res. 2014 Oct;8(10):DC01-3.

12. Patwardhan V, Kumar D, Goel V, Singh S. Changing prevalence and antibiotic drug resistance pattern of pathogens seen in community-acquired pediatric urinary tract infections at a tertiary care hospital of North India. J Lab Physicians. 2017;9(4):264-8.

13. Thaddanee R, Khilnani G, Shah N, Khilnani AK. Antibiotic sensitivity pattern of pathogens in children with urinary tract infection in a tertiary care hospital in Kachchh, Gujarat, India. Intern J Contemporary Pediatr. 2017 Oct 24;4(6):2103-8.

14. Taneja N, Chatterjee SS, Singh M, Singh S, Sharma M. Pediatric urinary tract infections in a tertiary care center from north India. Indian J Med Res. 2010 Jan 1;131(1):101-6.

15. Isolation and antibiotic susceptibility pattern of Escherichia coli from urinary tract infections in a tertiary care hospital of North Eastern Karnataka Kulkarni SR, Peerapur BV, Sailesh KS - J Nat Sc Biol Med. 2019. Available at: http://www.jnsbm.org/article.asp?issn=09769668; year $=2017$; volume $=8 ;$ issue $=2$; spage $=176$; epag e=180; aulast=Kulkarni. Accessed 11 June 2019.

16. Global prevalence of antibiotic resistance in paediatric urinary tract infections caused by Escherichia coli and association with routine use of antibiotics in primary care: systematic review and meta-analysis. 2019. Available at: https://www.ncbi.nlm.nih.gov/pmc/articles/PMC479 3155/. Accessed 11 June 2019.

Cite this article as: Angamuthu D, Bhaskar C, Aswathaman N. A retrospective study on prevalence, bacteriological profile and Antibiotic sensitivity pattern of Urinary Tract Infection in children of 2-12 years age in a tertiary care centre, Puducherry, India. Int J Contemp Pediatr 2019;6:1818-22. 